\title{
Theory of Three-Dimensional Nanocrescent Light
}

\section{Harvesters}

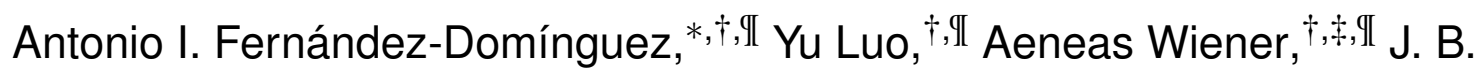 \\ Pendry, ${ }^{\dagger}$ and Stefan A. Maier ${ }^{\dagger}$ \\ Department of Physics, Imperial College London, London SW7 2AZ, and Department of \\ Materials, Imperial College London, London SW7 2AZ \\ E-mail: a.fernandez-dominguez@imperial.ac.uk
}

KEYWORDS: Localized surface plasmon, transformation optics, absorption cross section, field enhancement, structural bluntness, nonlocal effects

\begin{abstract}
The optical properties of three-dimensional crescent-shaped gold nanoparticles are studied using a transformation optics methodology. The polarization insensitive, highly efficient, and tunable light harvesting ability of singular nanocrescents is demonstrated. We extend our approach to more realistic blunt nanostructures, showing the robustness of their plasmonic performance against geometric imperfections. Finally, we provide analytical and numerical insights into the sensitivity of the device to radiative losses and nonlocal effects. Our theoretical findings reveal an underlying relation between structural bluntness and spatial dispersion in this particular nanoparticle configuration.
\end{abstract}

\footnotetext{
${ }^{*}$ To whom correspondence should be addressed

${ }^{\dagger}$ Department of Physics

$\ddagger$ Department of Materials

IIThese authors contributed equally
} 
Localized surface plasmons (LSPs) have the ability to beat the diffraction limit, which allows them to collect and concentrate light into deeply subwavelength volumes with high efficiency. This remarkable property, which is a clear manifestation of their hybrid electromagnetic-wave and surface-charge nature, is strongly dependent on the geometry, material characteristics, and environment of the metal nanoparticle sustaining them. The exploitation of the sensitivity of LSPs to all these circumstances has made possible an unprecedented control of light and light-matter interactions at the nanoscale. This fact has driven the emerging field of plasmonics, ${ }^{1}$ and has already yielded a wide range of novel LSP-based photonic devices. ${ }^{2,3}$

During the last number of years, intense research efforts have been devoted to devise, fabricate, and characterize plasmonic nanostructures with different functionalities. ${ }^{4}$ Among the profusion of designs investigated, dielectric core, metallic shell nanoparticles (usually termed nanoshells) have stood out for their extreme tunability. ${ }^{5}$ The appropriate choice of the inner dielectric material and the relative sizes of the core and the shell allows the tailoring of the of the LSPs supported by the structure within the whole optical spectrum. ${ }^{6,7}$ From an experimental side, these metallodielectric compounds have proven to be particularly accessible through a broad range of nanofabrication techniques. ${ }^{89}$ From a theoretical perspective, the optical properties of spherical core-shell geometries can be described accurately using Mie's theory, ${ }^{10}$ yielding a remarkable agreement with experimental observations. ${ }^{11,12}$ More recently, sophisticated numerical treatments beyond classical electromagnetics have been also developed. ${ }^{13,14}$ All these factors have made possible the realization of nanoshell plasmonic devices in technological areas as diverse as molecular sensing, ${ }^{15}$ cancer therapy ${ }^{16}$ and fluorescence microscopy. ${ }^{17}$

Nanoshells present a large effective cross section, which enables them to interact strongly with light. However, their LSP resonances do not yield to near-field hot spots as intense as those produced by structures with very small or sharp geometry features, such as dimer or trimer nanoantennas. ${ }^{18}$ Lately, a strategy to improve the focusing performance of nanoshells has been proposed. It consists in breaking the symmetry of the nanostructure by displacing the center of the dielectric core and the metal shell. ${ }^{19}$ Note that this also adds new structural degrees of freedom, increas- 
ing the tunability of the device. ${ }^{20-22}$ Experimental reports indicate that the nanoparticles resulting from this operation (usually termed nanoeggs or nanocups, depending on the inter-center distance relative to the shell radius) exhibit larger near-field enhancements than nanoshells, while retaining similar extinction efficiencies. ${ }^{23-25}$ So far, an analytical treatment of these reduced symmetry nanostructures is still lacking, and only numerical methods make a quantitative description of their optical properties possible. ${ }^{26,27}$ Moreover, whereas the hybridization concept ${ }^{28}$ yields a clear physical picture of the LSPs of the nanoshell, its insightfulness diminishes with increasing degree of structural asymmetry.

Recently, a transformation optics (TO) approach ${ }^{29}$ able to unveil hidden symmetries in complex plasmonic devices has been developed. The method was first devised to describe analytically the optical response of two-dimensional (2D) nanostructures, ${ }^{30,31}$ but it has been also successfully applied to the case of a dimer of touching nanospheres. ${ }^{32}$ In this Letter, we extend this threedimensional (3D) framework to crescent-shaped nanoparticles (nanocrescents). This geometry arises when the displacement of the dielectric core in a nanoshell is such that it leads to a single point where the metal thickness vanishes. The singular character of this geometry has prevented its rigorous treatment using hybridization or numerical methods thus far. ${ }^{19}$ On the contrary, our TO method yields analytical expressions for all the significant electromagnetic magnitudes in nanocrescents, revealing the efficient and tunable (polarization insensitive) light harvesting performance of the device over the whole optical spectrum.

By truncating singular 2D nanostructures, experimentally feasible geometries are obtained which can be described quasi-analitically using TO. ${ }^{33,34}$ This procedure is transferred to $3 \mathrm{D}$ in this Letter, demonstrating the robustness of blunt, nanocup-like plasmonic devices against structural defects. Our approach sheds light into the spectral discretization originating from edge rounding in nanocups, and proves that these non-singular structures retain the ability to collect and concentrate light efficiently. Finally, the radiative reaction concept ${ }^{35}$ provides us with analytical insight into the emergence of radiation losses as the nanostructure size becomes comparable to the incident wavelength. On the other hand, numerical calculations ${ }^{36,37}$ enable us to investigate nonlocal 

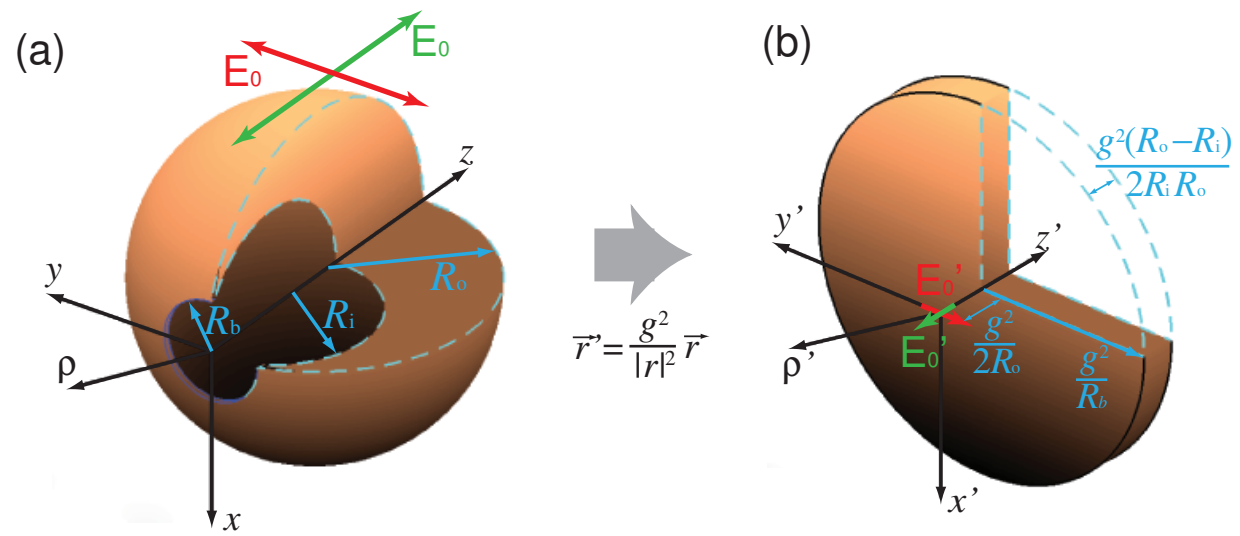

Figure 1: (a) Sketch of the problem under study, the optical properties of gold nanocrescents illuminated with arbitrarily polarized light. The geometry is defined by three parameters: the outer and inner radii of the gold shell, $R_{\mathrm{i}}$ and $R_{\mathrm{o}}$, respectively, and the bluntness radius, $R_{\mathrm{b}}$. (b) Disk geometry and dipole-like source obtained from the inversion of the original system.

effects in sub-nanometer thick shells, quantifying the impact of spatial dispersion on the absorption and field enhancement performance of the device. Our theoretical results show that bluntness and nonlocality modify the optical characteristics of perfect nanocrescents in a very similar way, revealing an underlying relation between these two distinct physical effects in this particular nanoparticle configuration.

Figure 1(a) shows the general problem under study, the interaction of arbitrarily polarized light with a crescent-shaped gold nanoparticle. The device is defined by three geometric parameters: $R_{\mathrm{i}}$ and $R_{\mathrm{O}}$, which correspond to the inner and outer radii of the asymmetric shell, respectively, and $R_{\mathrm{b}}$, which measures the structural bluntness. In the limit $R_{\mathrm{b}} \rightarrow 0$, a perfectly-shaped nanocrescent is obtained, which presents a singular vertex where the thickness of the gold shell vanishes. In any other case, this vertex is removed, and a hole of radius $R_{\mathrm{b}}$ is drilled in the metallic shell at this position. First, we restrict our attention to nanoparticles much smaller than the incoming wavelength $\left(R_{\mathrm{O}} \ll \lambda\right)$, which enables us to work within the near-field (quasi-static) approximation. Throughout this work, the gold permittivity, $\varepsilon_{M}(\omega)$, is modelled using experimental data. ${ }^{38}$

Figure 1(b) renders the structure and electromagnetic source obtained from the inversion of Figure 1(a), as indicated between the two panels. Note that unprimed (primed) coordinates denote 
original (transformed) coordinates, and $g$ is an arbitrary length constant. The transformation maps the original nanocrescent into a disk geometry of thickness $g^{2}\left(R_{\mathrm{O}}-R_{\mathrm{i}}\right) / 2 R_{\mathrm{O}} R_{\mathrm{i}}$ and radius $g^{2} / R_{\mathrm{b}}$. The disk radius becomes infinite under the inversion of a perfectly-shaped crescent $\left(R_{\mathrm{b}}=0\right)$. The incident fields convert into a dipole-like point source located at the origin of the inverted frame, and separated a distance $g^{2} / 2 R_{\mathrm{O}}$ from the disk. Contrary to its $2 \mathrm{D}$ counterpart, ${ }^{39}$ this operation does not only act on illumination and geometry, but also modifies the material properties of the gold structure and its environment. Thus, the permittivities in Figure 1(b) are not the original ones, but have acquired a spatial dependence proportional to $\left(g / r^{\prime}\right)^{2}$ under the inversion. ${ }^{32}$

The $r^{\prime}$-dependence of the inverted permittivities prevents the exact calculation of the quasistatic electric fields in the transformed frame. Specifically, fields continuity cannot be imposed at all points within the transformed disk surface. However, an approximate treatment of the problem is possible, and analytical expressions for all the relevant electromagnetic magnitudes in the crescent frame can be obtained. In the case of singular geometries $\left(R_{\mathrm{b}}=0\right)$, this procedure is carried out in three steps. First, fields continuity is imposed only at the crescent vertex $\left(\rho^{\prime} \rightarrow \infty\right.$ in the transformed frame). Second, only the surface plasmon pole contribution to the quasi-static fields is kept, neglecting the excitation of evanescent surface waves in the system. Finally, the mismatch of the displacement field at the nanocrescent surface far from the singularity is taken into account by introducing artificial surface charge corrections to the initial calculation. See the Supporting Information and Ref. 40 for a more detailed description of the solving strategy. This TO method yields the following expressions for the absorption cross section of perfect nanocrescents:

$$
\begin{aligned}
\sigma_{\mathrm{abs}}^{z} & =\frac{16 \pi \omega}{3 c}\left(\frac{2 R_{\mathrm{o}} \tau}{1-\tau}\right)^{3} \operatorname{Re}\left\{\frac{\varepsilon_{\mathrm{M}}(\omega) \alpha(\omega)}{1-\varepsilon_{\mathrm{M}}^{2}(\omega)}\left[2 \alpha(\omega)+\frac{1}{2 \tau}(1-\tau)\right] e^{-2 \alpha(\omega) \tau /(1-\tau)}\right\}, \\
\sigma_{\mathrm{abs}}^{y} & =\frac{32 \pi \omega}{3 c}\left(\frac{2 R_{\mathrm{o}} \tau}{1-\tau}\right)^{3} \operatorname{Re}\left\{\frac{\varepsilon_{\mathrm{M}}(\omega) \alpha^{2}(\omega)}{1-\varepsilon_{\mathrm{M}}^{2}(\omega)} e^{-2 \alpha(\omega) \tau /(1-\tau)}\right\},
\end{aligned}
$$

where Equations (1) and (2) correspond to axial (electric field parallel to z-direction) and normal (electric field perpendicular to $y$-direction) polarizations, respectively. Note that we have intro- 
duced the parameter $\tau=R_{\mathrm{i}} / R_{\mathrm{O}}$, which measures the gold shell thickness, and

$$
\alpha(\omega)=\frac{1}{2} \ln \left\{\frac{\varepsilon_{\mathrm{M}}(\omega)-1}{\varepsilon_{\mathrm{M}}(\omega)+1} \frac{\varepsilon_{\mathrm{M}}(\omega)-\varepsilon_{\mathrm{C}}}{\varepsilon_{\mathrm{M}}(\omega)+\varepsilon_{\mathrm{C}}}\right\},
$$

which governs the wavevector of the plasmonic modes supported by the nanocrescent. $\varepsilon_{\mathrm{C}}$ in Equation (3) stands for the dielectric constant filling the core of the nanoparticle. In contrast to 2D crescents, ${ }^{39}$ the absorption spectra for axial and normal illuminations present differences.

Figure 2(a) shows the absorption cross section of an air-filled perfect gold nanocrescent with $R_{\mathrm{O}}=10 \mathrm{~nm}$ and $\tau=0.85$ (note that more realistic dielectric cores are considered below). The left (right) panel renders $\sigma_{\text {abs }}$ under axial (normal) illumination. Solid lines plot analytical TO predictions, whereas dots correspond to numerical calculations. All the simulations presented in this work were performed using the highly adaptive meshing algorithm implemented in COMSOL Multiphysics. For comparison, the spectrum for a $10 \mathrm{~nm}$ radius nanosphere is also shown. Figure 2(a) demonstrates that nanocrescents present a larger and spectrally much broader cross section than solid nanoparticles. Our theoretical results are in good agreement with simulations at low frequencies, but overestimate $\sigma_{\mathrm{abs}}$ at frequencies near the crescent dipolar LSP resonance ( $\sim 450 \mathrm{THz})$. This is caused by the plasmon pole approximation intrinsic to Equations (1) and (2). Moreover, our TO approach also fails to reproduce the broad absorption band in the nanocrescent spectra above $500 \mathrm{THz}$, related to the emergence of interband transitions in the gold dielectric function.

Our TO method also yields analytical insight into the near-field characteristics of gold nanocrescents. The left inset of Figure 2(a) renders the $z$-component of the electric field, $E_{z}$, within the symmetry plane of the crescent at $300 \mathrm{THz}$ (axial polarization). It shows that the efficient absorption performance of the device originates from the excitation of plasmonic modes which propagate towards the vertex of the crescent. The group velocity of these electromagnetic waves decreases as they approach this geometric singularity, leading to an intense concentration of electromagnetic energy which is only truncated by metal absorption. Within the $x=0$ plane (see Figure 1 ), the TO 

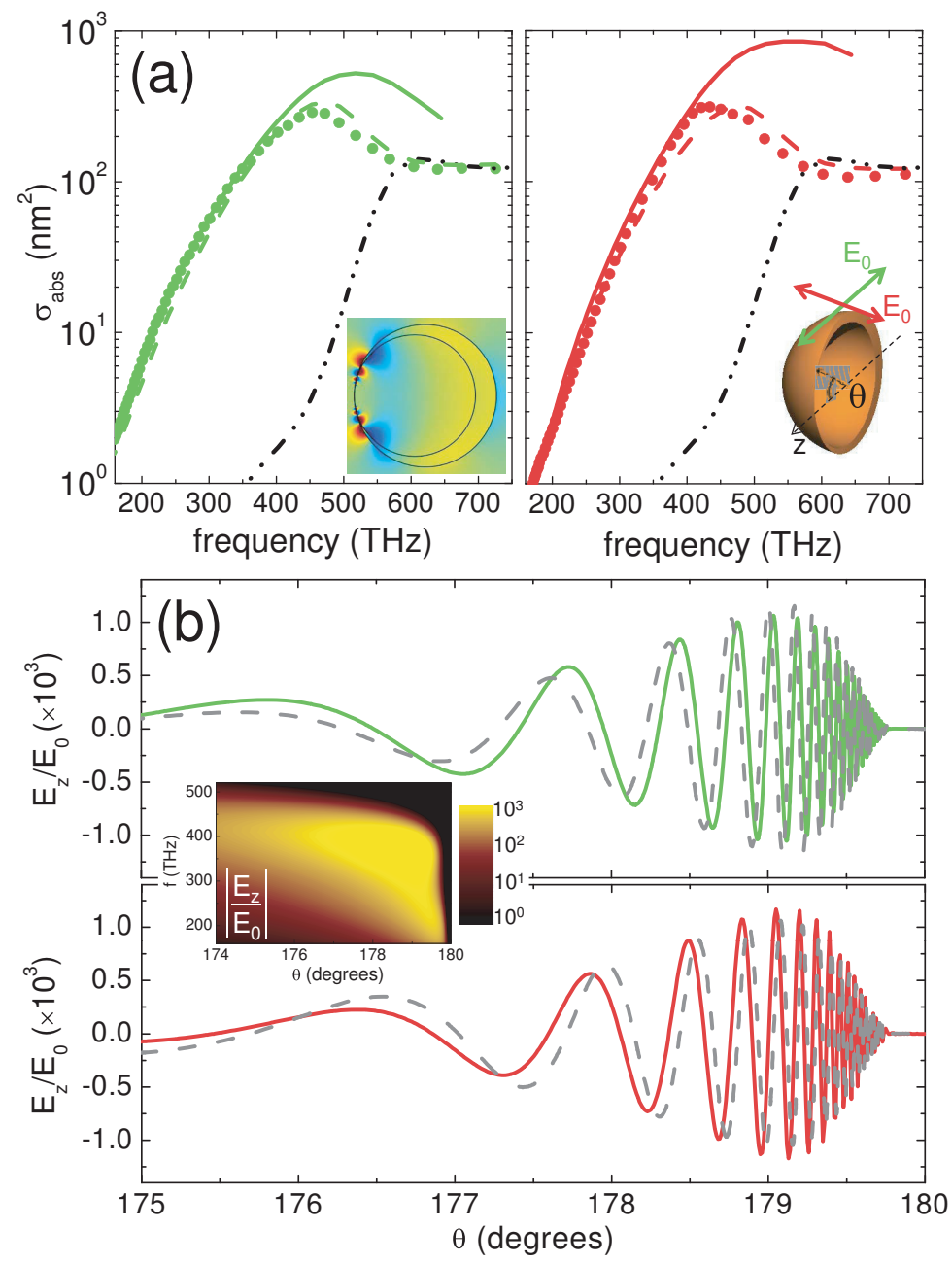

Figure 2: (a) Absorption cross section of a perfect gold nanocrescent $\left(R_{\mathrm{O}}=10 \mathrm{~nm}, \tau=0.85\right)$ under axial (left) and normal (right) illumination. Solid (dashed) lines render analytical (quasi-analytical) predictions, and dots correspond to numerical simulations. The solid sphere spectrum is plotted in black dashed-dotted line. The inset shows $E_{z} / E_{0}$ at $300 \mathrm{THz}$ in saturated colors ranging from -100 (blue) to 100 (red). (b) Field enhancement versus $\theta$ [see right inset in panel (a)] evaluated at $300 \mathrm{THz}$ for axial (top) and normal (bottom) polarization. Color solid (grey dashed) lines plot analytical (numerical) results. The inset renders $\left|E_{z} / E_{0}\right|$ versus the incident frequency and $\theta$ for both polarizations.

expression for the field enhancement in the vicinity of the nanocrescent vertex reads

$$
\begin{aligned}
\frac{E_{z}}{E_{0}}= & \sqrt{2} \pi\left(\frac{2 \tau}{1-\tau}\right)^{3} \frac{\alpha^{2}(\omega)}{1-\varepsilon_{\mathrm{M}}^{2}(\omega)} \frac{e^{-\alpha(\omega) \tau /(1-\tau)}}{(1+\cos \theta)^{3 / 2}} \times \\
& \times\left[\frac{\varepsilon_{\mathrm{M}}(\omega)+1}{\varepsilon_{\mathrm{C}}+1} e^{\alpha(\omega)}+\frac{\varepsilon_{\mathrm{M}}(\omega) \varepsilon_{\mathrm{C}}+1}{\varepsilon_{\mathrm{C}}+1}\right] J_{0,1}\left(\frac{\alpha(\omega) \tau}{1-\tau} \frac{\sin \theta}{1+\cos \theta}\right),
\end{aligned}
$$


where $J_{0}\left(J_{1}\right)$ denotes the zero (first) order Bessel function of the first kind, which must be used for the case of axial (normal) illumination. Equation (4) is evaluated at the external boundary of the nanoparticle, parameterized by the angle $\theta$ as indicated in the right inset of Figure 2(a). Note that the electric field distribution is azimuthally independent for incident polarization parallel to the crescent axis.

Figure 2(b) plots the field enhancement in the vicinity of the nanocrescent singularity $\left(R_{\mathrm{O}}=10\right.$ $\mathrm{nm}, \tau=0.85$ ) for axial (top) and normal (bottom) illumination (300 THz). Color solid lines render $E_{z} / E_{0}$ obtained from Equation (4), whereas numerical calculations are shown in grey dashed lines. Theory and simulations are in remarkable agreement (except for a small angular shift), which validates the physical approximations behind our TO calculation. The inset of Figure 2(b) displays $\left|E_{z} / E_{0}\right|$ as a function of the incident frequency and the angle $\theta$. Interestingly, the theoretical prediction shows that the electric fields for both polarizations have the same magnitude, revealing the polarization independent nanofocusing ability of 3D nanocrescents. This effect can be easily understood through Equation (4), as the asymptotic forms of the Bessel functions at the crescent vertex $\left(\theta \rightarrow 180^{\circ}\right)$ differ only in a phase term. The field enhancement contourplot illustrates the balance between geometric focusing and absorption damping taking place in the nanostructure. At low frequencies, the large metal permittivity and small absorption lead to a strong localization of the electromagnetic energy at the crescent singularity. On the other hand, the electric fields are damped at positions further from this point with increasing frequency. This effect not only shifts the maximum in $\left|E_{z} / E_{0}\right|$ to smaller $\theta$, but also widens its angular extension. Importantly, our TO calculations indicate that the value of the maximum field enhancement $\left(\sim 10^{3}\right)$ is independent of the incident frequency below the dipolar LSP.

Up to here, we have described the ability of gold crescents to convert radiation from the far to the near-field with high efficiency within a broad frequency window. We investigate now the tuning possibilities of the nanostructure. Taking advantage of the insensitivity of the device performance to the incident polarization, we restrict our analysis to axial illumination in the following (see the Supporting Information for more details on normal illumination). Figure 3(a) explores the 

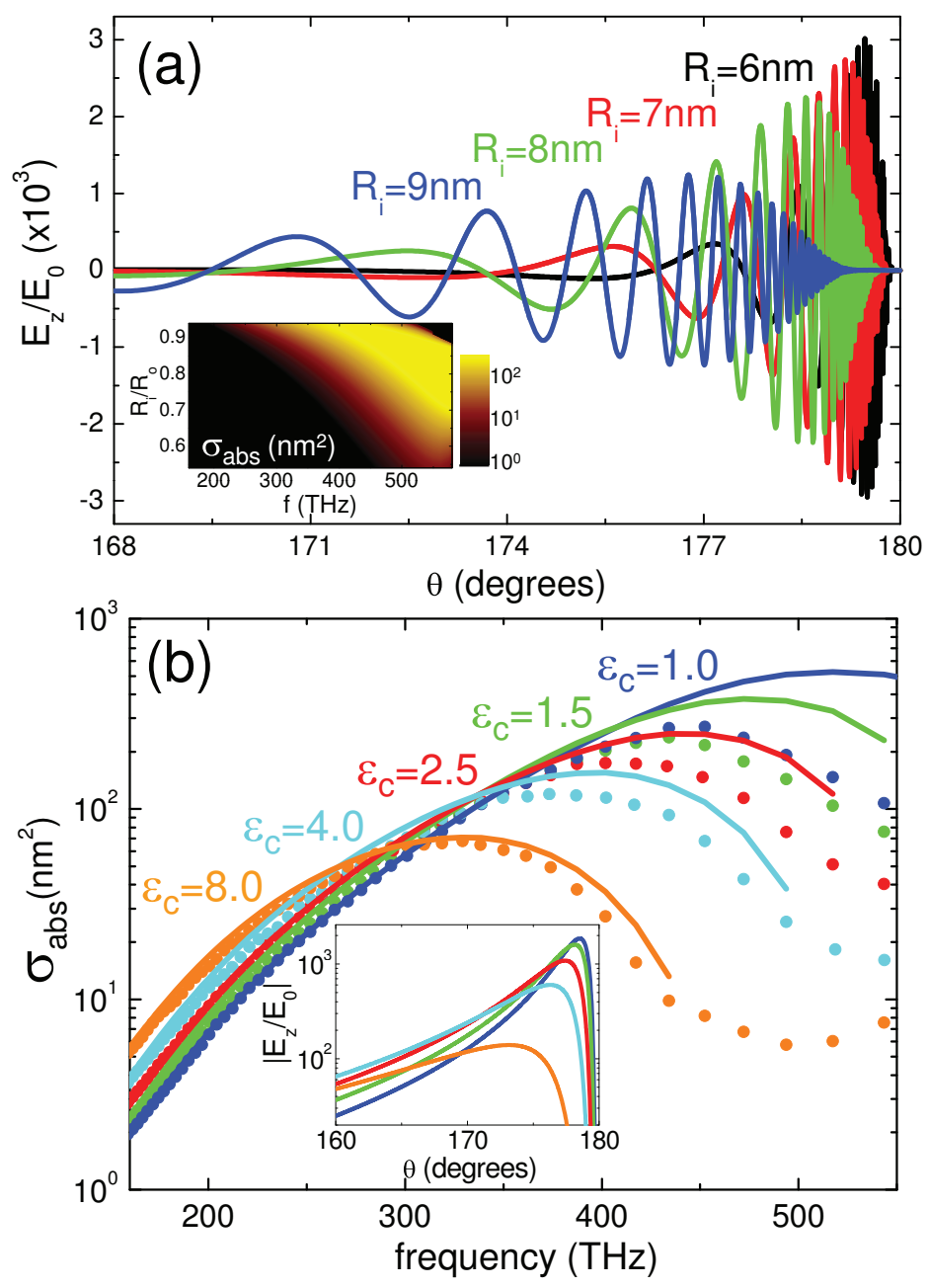

Figure 3: (a) Electric field enhancement versus $\theta$ evaluated at $300 \mathrm{THz}$ for air-filled nanocrescents with $R_{\mathrm{O}}=10 \mathrm{~nm}$ and different $R_{\mathrm{i}}$. The inset renders $\sigma_{\mathrm{abs}}$ as a function of the incident frequency and $\tau$ in $\log$ scale. (b) Absorption spectra for crescents $\left(R_{\mathrm{O}}=10, \tau=0.85\right)$ filled with different dielectric cores. Solid lines (dots) correspond to analytical (numerical) results. The inset plots $\left|E_{z} / E_{0}\right|$ versus $\theta$ at $300 \mathrm{THz}$ for the geometries in the main panel. 
dependence of the crescent nanofocusing ability on the shell thickness. Theoretical $E_{z} / E_{0}$ at 300 THz is plotted versus the angle $\theta$ for $R_{\mathrm{O}}=10 \mathrm{~nm}$ nanoparticles of various $\tau$. The thickening of the gold cover (reduction of $R_{\mathrm{i}}$ ) increases the field localization at the crescent singularity, which also leads to higher $E_{z} / E_{0}$ values. The inset of Figure 3(a) renders $\sigma_{\mathrm{abs}}$ as a function of the frequency and $\tau$. It shows the red-shifting of the absorption maximum with the shell thinning. This trend can be understood in terms of the transformed disk, whose thickness is proportional to $(1-\tau) / \tau$. Crescents with larger $\tau$ map into thinner disks, which increases the binding (and therefore lowers the frequency) of the surface plasmon modes supported by the system.

Figure 3(b) shows the absorption spectra for nanoparticles with $R_{\mathrm{O}}=10 \mathrm{~nm}, \tau=0.85$, and different dielectric cores, $\varepsilon_{\mathrm{C}}$. The TO predictions given by Equation (1) (solid lines) are in very good agreement with numerical results (dots). We can observe that increasing $\varepsilon_{\mathrm{C}}$ has a similar effect on the cross section as increasing $\tau$. This can be interpreted again as a consequence of the tightening of the plasmonic modes in the inverted structure due to the presence of a higher dielectric environment. Note that the crescent core maps into the semi-infinite volume $z^{\prime}>g^{2} / R_{\mathrm{i}}$ in the transformed frame. The spectra in Figure 3(b) also show a notable reduction of $\sigma_{\mathrm{abs}}$ just above the absorption maxima. The origin of this effect can be clearly identified in Equation (3). At frequencies satisfying $-\varepsilon_{\mathrm{C}}<\varepsilon_{\mathrm{M}}(\omega)<-1$, the real part of $\alpha(\omega)$ (which gives the surface plasmon propagating wavevector) vanishes. This means that the gold nanocrescents do not support plasmonic modes able to collect the incident light within this spectral window, which causes their small effective cross section. The inset of Figure 3(b) plots $\left|E_{z} / E_{0}\right|$ at $300 \mathrm{THz}$ in the vicinity of the crescent singularity. It demonstrates the tunability of the light concentration profile by means of the dielectric medium filling the core. Finally, we remark that the dependence of the spectral characteristics of nanocrescents on $\tau$ and $\varepsilon_{\mathrm{C}}$ makes their tunability throughout the whole visible spectrum possible.

Perfectly-shaped nanocrescents are an idealization of the actual nanoparticle designs achievable experimentally. Although their analytical study has provided us with deep physical insight, we focus our attention now on more feasible nanostructures. Specifically, we address the question 
of how the light harvesting capabilities of these devices are modified when their singular vertex is removed. As shown in Figure 1(a), we consider the blunt geometry that results from the drilling of a circular hole of radius $R_{\mathrm{b}}$ at the crescent vertex (note that the spherical inner core remains unaltered). Such nanocup-like structures have been realized experimentally using various nanofabrication techniques. ${ }^{21,23}$

Our TO approach enables us to describe the optical properties of blunt nanocrescents or nanocups. As discussed above, these lead to disks of finite radius in the transformed frame, see Figure 1(b). Contrary to perfect crescents, nanocups cannot be linked to infinite plasmonic structures, which implies that they do not exhibit the continuous response of the singular geometry. Thus, the electric fields for blunt nanocrescents cannot be expressed in terms of a single plasmon pole contribution, but as a superposition of standing surface waves. Under the inversion, surface plasmon modes are excited by the transformed dipole-like source and propagate away within the flat disk faces. These are perfectly reflected at the disk edges (re-irradiation effects are neglected within the near-field approximation), and travel back and forth until being damped due to metal absorption. Therefore, due to the finite size of its transformed counterpart, the surface plasmon modes supported by nanocups are no longer continuous, but discrete at different resonant frequencies. Using the quasianalytical TO approach summarized in the Supporting Information, the absorption cross section for blunt nanocrescents can be written as

$$
\sigma_{\mathrm{abs}}^{z}=\frac{4 \pi \omega}{c} R_{\mathrm{o}}^{3} \operatorname{Im}\left\{\sum_{l=0}^{l_{\max }} \alpha_{l} B_{l}\right\}
$$

where $\alpha_{l}$ corresponds to the $l$-th zero of the zero order Bessel function and $B_{l}$ is a coefficient calculated by appropriately applying boundary conditions at several discrete points along the crescent surface. The parameter $l_{\max }$ corresponds to the number of terms needed in Equation (5) to reach convergence $\left(\sim 10-50\right.$, increasing with smaller $\left.R_{\mathrm{b}}\right)$. In the Supporting Information, the derivation of the cross section for normal illumination is also provided. The color dashed lines in Figure 2(a) plot the spectra obtained from Equation (5) for perfect crescents. They show that 
(a)

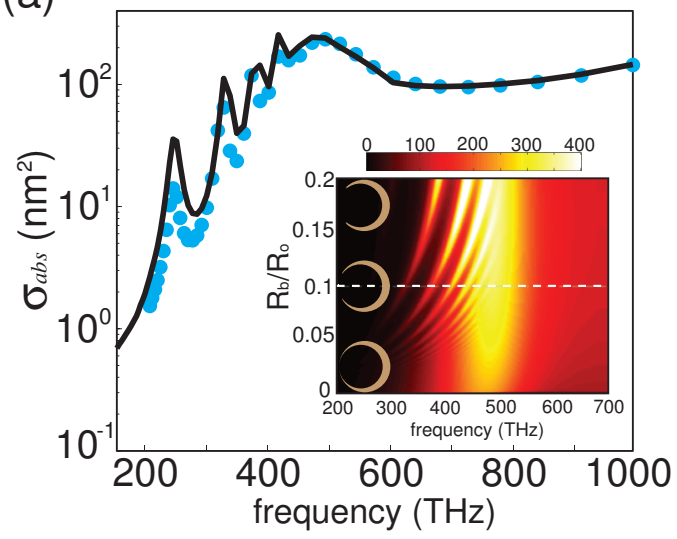

(b)

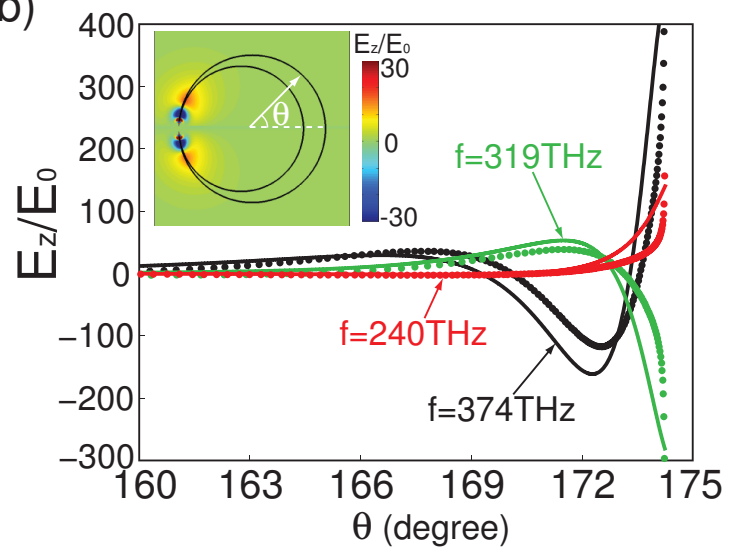

Figure 4: (a) Quasi-analytical TO (solid line) and numerical (dots) absorption cross section for a blunt nanocrescent with $R_{\mathrm{O}}=10 \mathrm{~nm}, \tau=0.85$, and $R_{\mathrm{b}}=1 \mathrm{~nm}$. The inset renders $\sigma_{\mathrm{abs}}$ in $\mathrm{nm}^{2}$ as a function of the incident frequency and the ratio $R_{\mathrm{b}} / R_{\mathrm{O}}$ for the $R_{\mathrm{O}}$ and $\tau$ values in the main panel. (b) $E_{z} / E_{0}$ versus $\theta$ for the three lowest absorption maxima in panel (a). Solid lines (dots) correspond to TO (numerical) calculations. The inset shows the field enhancement within the crescent middle plane at $374 \mathrm{THz}$.

our quasi-analytical predictions are in excellent agreement with simulations even above the dipolar LSP resonance. This is due to the fact that they are no longer based on the plasmon pole approximation, and have taken into account the contribution from all electromagnetic modes, including evanescent surface waves.

The main panel of Figure 4(a) plots the TO quasi-analytical (solid line) and numerical (dots) absorption cross section for a blunt crescent with $R_{\mathrm{O}}=10 \mathrm{~nm}, \tau=0.85$, and $R_{\mathrm{b}}=1 \mathrm{~nm}$. The spectra display four distinct resonant peaks below the dipolar LSP, resulting from the plasmonic mode discretization described above. For larger bluntness radii, the intensity of these absorption maxima increases while their spectral position blue-shifts. This is illustrated in the inset of Figure 4(a), which renders $\sigma_{\mathrm{abs}}$ in $\mathrm{nm}^{2}$ versus the incident frequency and the ratio $R_{\mathrm{b}} / R_{\mathrm{O}}$. For small bluntness $\left(R_{\mathrm{b}}<0.02 R_{\mathrm{O}}\right)$, the absorption spectra exhibit the continuous broadband behaviour characteristic of singular nanocrescents. For larger truncations, a well defined array of resonances appear, which govern the light collection properties of nanocups when $R_{\mathrm{b}}$ and $R_{\mathrm{O}}$ are comparable.

In order to gain physical insight into the plasmonic resonances arising in the absorption spec- 

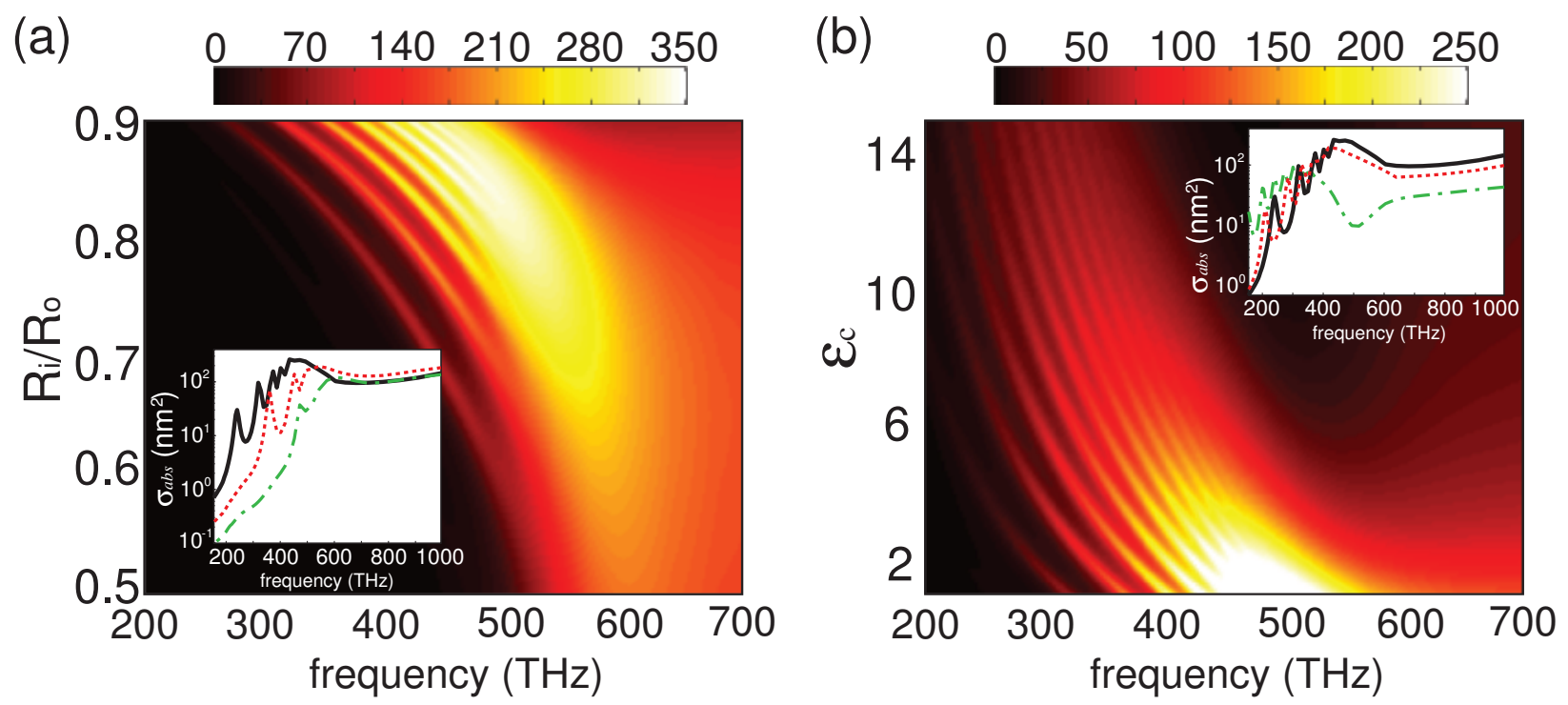

Figure 5: (a) Quasi-analytical absorption cross section as a function of the incident frequency and $\tau=R_{\mathrm{i}} / R_{\mathrm{O}}$ for nanocups with $R_{\mathrm{O}}=10 \mathrm{~nm}, R_{\mathrm{b}}=1 \mathrm{~nm}$, and $\varepsilon_{\mathrm{C}}=1$. The inset shows the spectra for $\tau=0.5$ (green), 0.7 (red) and 0.85 (black). (b) $\sigma_{\text {abs }}$ as a function of the incident frequency and the dielectric core for blunt crescents with $R_{\mathrm{O}}=10 \mathrm{~nm}, \tau=0.85$ and $R_{\mathrm{b}}=1 \mathrm{~nm}$. The inset shows the absorption spectra for different $\varepsilon_{\mathrm{C}}: 1$ (black), 2 (red) and 12 (green).

trum of gold nanocups, we analyze now the nanofocusing performance of these devices. Figure 4(b) plots $E_{z} / E_{0}$ versus $\theta$ (see inset) at the three lowest absorption maxima for the nanocrescent considered in panel (a). Both theoretical and numerical results are shown, in solid lines and dots, respectively. The electric field profiles demonstrate that each plasmonic mode features a distinct number of spatial oscillations along the nanocrescent surface. This is in excellent agreement with the standing wave picture introduced above. Note that for the three resonances, the maximum of $E_{z} / E_{0}$ takes place at the nanocup edge. Importantly, although the structure truncation diminishes the focusing efficiency, field enhancements up to 400 are obtained for the geometry considered in Figure 4. The inset of Figure 4(b) renders $E_{z} / E_{0}$ within the crescent middle plane evaluated at 374 THz. See the Supporting Information for a similar study on nanocups under normal illumination.

Figure 5(a) investigates the dependence of the absorption properties of blunt nanocrescents on the thickness of the gold shell. It renders $\sigma_{\text {abs }}\left(\right.$ in $\mathrm{nm}^{2}$ ) versus the incident frequency and the parameter $\tau$ for $10 \mathrm{~nm}$ outer radius nanocups with $R_{\mathrm{b}}=1 \mathrm{~nm}$ and $\varepsilon_{\mathrm{C}}=1$. The contourplot indicates that the sensitivity of the absorption behavior against structural imperfections diminishes 
with increasing thickness of the gold shell (decreasing $\tau$ ). For thin nanocrescents, the cross section displays multiple high intensity resonances, which emerge already at low frequencies. The shell thickening shifts these resonances towards the dipolar LSP $(\sim 450 \mathrm{THz})$, where all merge for $\tau<0.6$. This trend is accompanied by an overall reduction of the absorption cross section, as evidenced in the inset of Figure 5(a). The absorption spectrum for three shell thicknesses is plotted, $\tau=0.5$ (green), 0.7 (red) and 0.85 (black), demonstrating that the disappearance of the discrete surface plasmon resonances results in the degeneration of the nanocrescent cross section to the spectrum of a solid nanosphere. Thus, in order to optimize the light-harvesting performance of 3D gold nanocups, the shell thickness must be appropriately designed. It should be neither too small (in order to achieve a considerable field enhancement) nor too large (so that the structure supports LSP resonances at the operation window). For instance, for a nanocup with a 10nm outer and a $1 \mathrm{~nm}$ bluntness radii, our theoretical approach predicts that the optimum shell thickness range is $0.7<\tau<0.85$.

An additional degree of freedom is introduced in Figure 5(b), which explores the variation of $\sigma_{\mathrm{abs}}$ with the dielectric constant filling the core of nanocups with $R_{\mathrm{o}}=10 \mathrm{~nm}, \tau=0.85$ and $R_{\mathrm{b}}=1$ $\mathrm{nm}$. It is apparent that increasing $\varepsilon_{\mathrm{C}}$ results in a red-shifting of the absorption maxima, which is similar to the perfect crescent behaviour observed in Figure 3(b). This effect is accompanied by the suppression of the continuous absorption band above $500 \mathrm{THz}$, which again can be explained in terms of the frequency window in which $\operatorname{Re}\{\alpha(\omega)\}=0$ in Equation (3). As a result of the reduction of $\sigma_{\mathrm{abs}}$ above $500 \mathrm{THz}$, the discrete absorption resonances shift to lower frequencies, while their intensity reduces. This trend can be clearly seen in the inset of Figure 5(b), which plots the cross section for nanocups with $\varepsilon_{\mathrm{C}}=1$ (black), 2 (red) and 12 (green). Calculations for normal incident polarization are provided in the Supporting Information, showing a similar behaviour.

Radiation losses emerge for crescent dimensions comparable to the incident wavelength. In order to describe this additional damping channel in 3D nanostructures, we must go beyond the near-field approximation. With this purpose, we use here a self-consistent methodology based on the optical theorem. Detailed calculations (described in the Supporting Information) show that the 


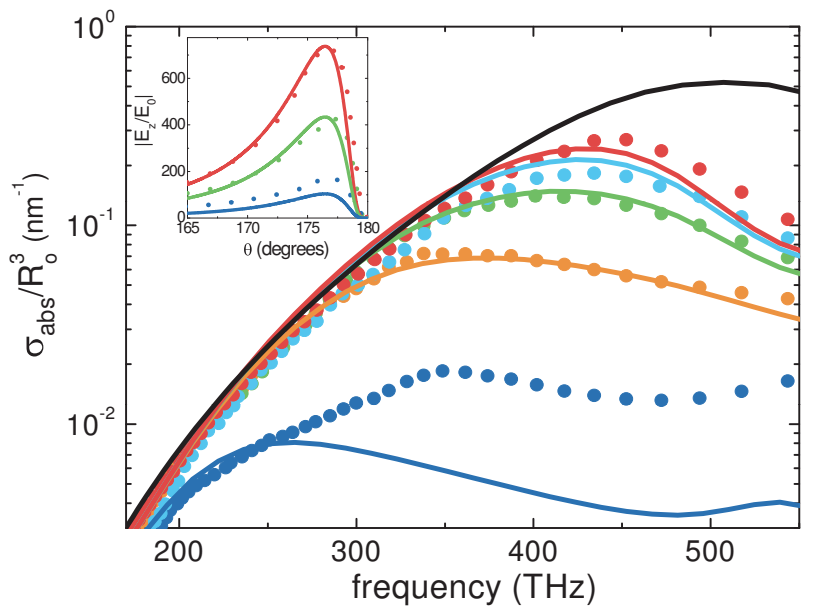

Figure 6: Quasi-analytical (solid lines) and numerical (dots) absorption cross section normalized to the nanoparticle volume $\left(R_{\mathrm{o}}^{3}\right)$ for singular crescents with $\tau=0.9$ and different outer radii: 10 $\mathrm{nm}$ (red), $30 \mathrm{~nm}$ (cyan), $50 \mathrm{~nm}$ (green), $100 \mathrm{~nm}$ (orange), and $200 \mathrm{~nm}$ (blue). The analytical TO prediction is shown in black solid line. The inset shows $\left|E_{z} / E_{0}\right|$ versus $\theta$ evaluated at $400 \mathrm{THz}$ for perfect crescents with outer radius as indicated by the color code in the main panel.

nanocrescent cross section corrected in this way takes the following form

$$
\sigma_{\mathrm{abs}}^{z}=\frac{4 \pi \omega}{\eta^{2}(\omega) c} R_{\mathrm{o}}^{3} \operatorname{Im}\left\{\sum_{l=0}^{l_{\max }} \alpha_{l} B_{l}\right\}
$$

where $\eta(\omega)=\left|1-\frac{2 i}{3}\left(\frac{\omega}{c}\right)^{3} R_{\mathrm{o}}^{3} \sum_{l=0}^{l_{\max }} \alpha_{l} B_{l}\right|$ is the so-called radiative reaction factor. ${ }^{35}$ Note that the second term in $\eta(\omega)$ describes the occurrence of radiative damping. When the structure dimensions are small, $\omega R_{\mathrm{O}} / c \ll 1$, and this term vanishes. In this limit, Equation (6) reduces to the quasi-static expression.

Figure 6 depicts the normalized-to-volume absorption cross section for singular crescents with different outer radii, $R_{\mathrm{o}}$ : 10 (red), 30 (cyan), 50 (green), 100 (orange), and 200 (blue) nm. The good agreement between the quasi-analytical and numerical results for $R_{\mathrm{O}} \leq 100 \mathrm{~nm}$ demonstrates the validity of our TO approach. When $R_{\mathrm{O}} \geq 200 \mathrm{~nm}$, our theoretical predictions differ from the numerical simulations due to the appearance of the retardation effects that have been ignored in our treatment. As indicated by Figure 6, the absorption behavior of the 3D crescent remains insensitive to size effects for $R_{\mathrm{O}} \leq 50 \mathrm{~nm}$. Note that the black solid line plots the analytical normalized spectrum obtained from Equation (1), which is independent of the nanocrescent outer radius. For 
(a)

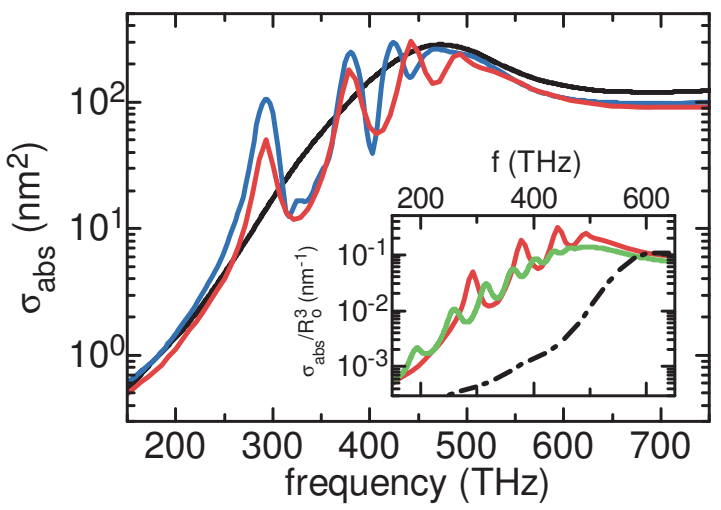

(b)

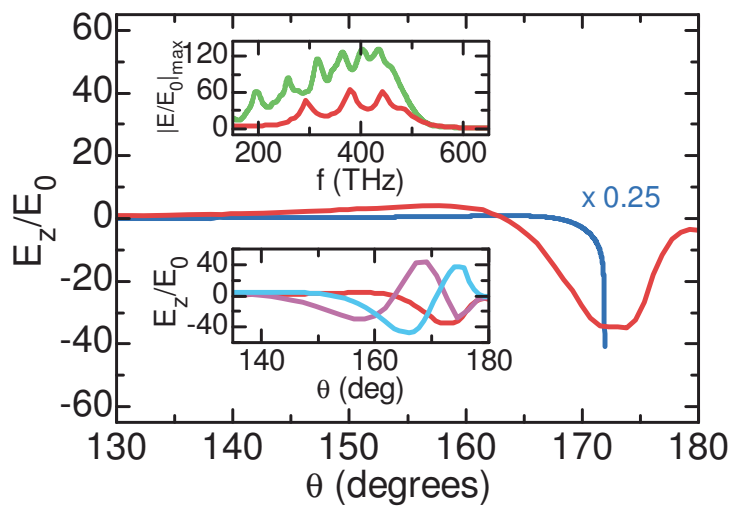

Figure 7: (a) Absorption cross section of gold nanocrescents with $R_{\mathrm{O}}=10 \mathrm{~nm}$ and $\tau=0.85$. The red (blue) line renders the nonlocal (local) spectrum for the singular (blunt) geometry with $R_{\mathrm{b}}=0$ $\left(R_{\mathrm{b}}=1.4 \mathrm{~nm}\right)$. The singular local cross section is plotted in black line. The inset shows nonlocal $\sigma_{\mathrm{abs}} / R_{\mathrm{o}}^{3}$ for singular crescents with 10 (red) and 40 (green) $\mathrm{nm}$ outer radius, together with the nonlocal spectrum for a $10 \mathrm{~nm}$ radius solid nanosphere (black). (b) Field enhancement versus $\theta$ for the lowest absorption maximum $(290 \mathrm{THz})$ in the nonlocal (red) and blunt (blue) spectra in (a). The lower inset shows $E_{z} / E_{0}$ for the three lowest nonlocal resonances in (a). The upper panel plots the maximum $\left|E_{z} / E_{0}\right|$ versus frequency for $R_{\mathrm{o}}=10$ (red) and 40 (green) nm perfect crescents within the nonlocal approximation.

larger dimensions, radiative damping reduces $\sigma_{\mathrm{abs}}$, while shifting to lower frequencies the cross section maximum. The inset of Figure 6 shows the electric field enhancement at $400 \mathrm{THz}$ for crescents with $R_{\mathrm{O}}$ as indicated by the color code in the main panel. Dots plot numerical calculations, and solid lines render $\left|E_{z} / E_{0}\right|$ obtained from Equation (4), divided by the radiative correction factor $\eta(\omega)$. The field enhancement maximum in Figure 6 only decreases by a factor of 2 when $R_{\mathrm{O}}$ increases from $10 \mathrm{~nm}$ to $50 \mathrm{~nm}$. This indicates that $3 \mathrm{D}$ nanocrescents are much more robust to radiative losses than their $2 \mathrm{D}$ counterparts. ${ }^{39}$

So far, we have treated the optical properties of gold crescents within the usual local description for the metal permittivity. However, recent studies ${ }^{41-44}$ demonstrate the emergence of significant quantum phenomena in plasmonic devices shaped on sub-nanometer length scales. Specifically, nonlocal effects in the metal dielectric function give rise to an effective smearing out of the nanostructure boundaries. This reduces the binding of LSP modes and diminishes the achievable field enhancements. ${ }^{36,45}$ Here, we investigate the impact that nonlocality has on the absorption and 
focusing performance of nanocrescents using a quasi-3D, spatially dispersive, finite element numerical method (see the Supporting Information for further details).

Figure 7(a) compares the cross section of a singular crescent $\left(R_{\mathrm{O}}=10 \mathrm{~nm}, \tau=0.85\right)$ treated within the nonlocal description (red line) and the local approximation (black line). The introduction of spatial dispersion leads to the emergence of discrete resonant peaks which are reminiscent of those found for blunt geometries (see Figure 4). The blue line in Figure 7(a) renders the local absorption cross section for a nanocup with $R_{\mathrm{b}}=1.4 \mathrm{~nm}$. Importantly, the bluntness radius has been chosen so that the metal thickness at the cup edge is twice the longitudinal plasmon decay length $\left(\delta_{\mathrm{LP}}=0.1 \mathrm{~nm}\right.$ for gold). These two spectra (red and blue lines) present not only a qualitative, but a quantitative resemblance, which gives us physical insight into the impact that spatial dispersion has on the device. This indicates that the surface plasmon modes travelling towards the nonlocal crescent vertex undergo reflection when reaching shell regions thinner than $2 \delta_{\mathrm{LP}}$. Within the nonlocal description, the surface plasmon modes lose their ability to accommodate to the metal boundaries in the sub-nanometer regime. As radiation losses are negligible for small nanoparticles, these modes suffer reflection in a similar way as it happens in truncated geometries.

Our interpretation of spatial dispersion in gold nanocrescents is supported by the inset of Figure 7(a). It compares nonlocal $\sigma_{\mathrm{abs}} / R_{\mathrm{o}}^{3}$ for singular crescents $(\tau=0.85)$ with $R_{\mathrm{O}}=10$ (red) and 40 (green) nm. Similarly to the nanocups in Figure 5, Figure 7(a) indicates that bigger nanocrescents develop a larger number of less defined nonlocal resonances. This can be linked to the effective expansion of the shell area where the gold thickness is comparable to $\delta_{\mathrm{LP}}$. For $R_{\mathrm{O}}>40 \mathrm{~nm}$, the nonlocal maxima blur and a continuum spectrum is recovered. Importantly, both nonlocal crescents exhibit a larger and broader cross section than the $10 \mathrm{~nm}$ radius nonlocal nanosphere plotted in black dashed line.

We analyze in Figure 7(b) the impact of nonlocality on the field enhancement ability of gold crescents. The main panel shows $E_{z} / E_{0}$ versus $\theta$ for the lowest resonance $(290 \mathrm{THz})$ in the nonlocal (red) and blunt (blue) spectra in Figure 7(a). The electric field associated to the plasmonic modes supported by both crescents changes sign only once as they approach $\theta=180^{\circ}$. Moreover, 
the maximum field enhancement takes place at the same position $\left(\theta=172^{\circ}\right)$ in both cases. This allows us to extend the link between nonlocal and bluntness effects discussed above from the far to the near field. Note that the maximum in $E_{z} / E_{0}$ is 4 times larger for the blunt nanocup than for the nonlocal crescent. This can be related to the lighting rod effect, ${ }^{1}$ which does not occur in the singular geometry. The lower inset of Figure 7(b) compares $E_{z} / E_{0}$ for the three lowest nonlocal resonances in Figure 7(a). Whereas the lowest resonance oscillates once in the vicinity of the crescent vertex (red), the second (blue) and third (violet) present two and three oscillations, respectively.

Finally, we investigate to which degree nonlocal effects and radiative losses limit the field enhancement that can be achieved in nanocrescents. Whereas the former govern the optical properties of small devices, the latter become relevant in large ones. The main panel of Figure 7(b) demonstrates that, once spatial dispersion is taken into account, the field enhancement in a $10 \mathrm{~nm}$ outer radius crescent at its lowest resonance reaches a maximum value of 34 . On the other hand, the inset of Figure 6 indicates that radiation effects are not significant for $R_{\mathrm{O}} \leq 50 \mathrm{~nm}$. The nanofocusing performance of the device should be optimized for dimensions between these two cases. This is shown in the upper panel of Figure 7(b), which plots the maximum $\left|E_{z} / E_{0}\right|$ as a function of frequency for the nonlocal crescents in the inset of Figure 7(a). Note that the angular position of $\left|E_{z} / E_{0}\right|_{\max }$ shifts with frequency. Importantly, whereas the maximum field enhancement for $R_{\mathrm{O}}=10 \mathrm{~nm}$ (red) is $60,\left|E_{z} / E_{0}\right|_{\max }=130$ is predicted for $40 \mathrm{~nm}$ outer radius nanocrescents (green).

Summarizing, we have introduced a set of highly versatile TO solutions revealing the electromagnetic properties of 3D nanocrescents. Our approach incorporates both bluntness effects, inevitable in any nanofabrication strategy, as well as radiative damping, significant in devices whose dimensions lead to the scattering of electromagnetic energy into the far field. Applying these methods, we have obtained deep insights into how geometric and material aspects govern the performance of crescent-shaped light harvesters. Finally, a spatially dispersive treatment of Maxwell's equations was used to determine the impact of nonlocal effects in subnanometer shells. 
Crucially, we find that, despite adverse effects such as structural imperfections, radiation losses, or nonlocality, field enhancements of more than two orders of magnitude take place in realistic gold nanocrescent designs.

\section{Acknowledgement}

This work was sponsored by the EU project PHOME (Contract No. 213390), the ESF PlasmonBionanosense Network, the Leverhulme Trust, and the Engineering and Physical Sciences Research Council (EPSRC). The authors thank Andrew P. Horsfield for useful discussions. A. W. was supported by the Doctoral Training Centre in Theory and Simulation of Materials. Y. L. acknowledges the Lee family scholarship for financial support.

\section{References}

(1) Maier, S. A. Plasmonics: Fundamentals and Applications; Springer Verlag, 2007.

(2) Atwater, H. A.; Polman, A. Nature Materials 2010, 9, 205-213.

(3) Novotny, L.; van Hulst, N. Nature Photonics 2011, 5, 83-90.

(4) Giannini, V.; Fernández-Domínguez, A. I.; Heck, S. C.; A, M. S. Chemical Reviews 2011, $111,3888-3912$.

(5) Halas, N. MRS Bulletin 2005, 30, 362-367.

(6) Tam, F.; Moran, C.; Halas, N. J. J. Phys. Chem. B 2004, 108, 17290-17294.

(7) Bardhan, R.; Grady, N. K.; Ali, T.; Halas, N. J. ACS Nano 2010, 4, 6169-6179.

(8) Sun, Y.; Mayers, B.; Younan, X. Advanced Materials 2003, 15, 641-646.

(9) Radt, B.; Smith, T. A.; F, C. Advanced Materials 2004, 16, 2184-2189.

(10) Aden, A. L.; Kerker, M. J. Appl. Phys. 1951, 22, 1242-1246. 
(11) Averitt, R. D.; Westcott, S. L.; Halas, N. J. J. Optl. Am. B 1999, 16, 1824-1832.

(12) Nehl, C. L.; Grady, N. K.; Goodrich, G. P.; Tam, F.; Halas, N. J.; Hafner, J. H. Nano Letters 2004, 4, 2355-2359.

(13) Prodan, E.; Nordlander, P.; Halas, N. J. Nano Letters 2003, 3, 1411-1415.

(14) Baer, R.; Neuhauser, D.; Weiss, S. Nano Letters 2004, 4, 85-88.

(15) Raschke, G.; Brogl, S.; Shusa, A. S.; Rogach, A. L.; Klar, T. A.; Feldmann, J.; Fieres, B.; Petkov, N.; Bein, T.; Nichtl, A.; Kürzinger, K. Nano Letters 2004, 4, 1853-1857.

(16) Hirsch, L. R.; Stafford, R. J.; Bankson, J. A.; Sershen, S. R.; Rivera, B.; Price, R. E.; Hazle, J. D.; Halas, N. J.; West, J. L. Proceedings of the National Academy of Sciences of the United States of America 2003, 100, 13549-13554.

(17) Sivan, Y.; Sonnefraud, Y.; Kena-Cohen, S.; Pendry, J. B.; A, M. S. ACS Nano 2012, 6, 52915296.

(18) Koh, A. L.; Fernández-Domínguez, A. I.; McComb, D. W.; Maier, S. A.; W, Y. J. K. Nano Letters 2011, 11, 1323-1330.

(19) Wang, H.; Wu, Y.; Lassiter, B.; Nehl, C. L.; Hafner, J. H.; Nordlander, P.; Halas, N. J. Proceedings of the National Academy of Sciences of the United States of America 2006, 103, 10856-10860.

(20) Bukasov, R.; Shumaker-Parry, J. S. Nano Letters 2007, 7, 1113-1118.

(21) Lassiter, J. B.; Knight, M. W.; Mirin, N. A.; Halas, N. J. Nano Letters 2009, 9, 4326-4332.

(22) Huang, F. M.; Wilding, D.; Speed, A. E., J D abd Russell; Bartlett, P. N.; Baumberg, J. J. Nano Letters 2011, 11, 1221-1226.

(23) Lu, Y.; Liu, G. L.; Kim, J.; Mejia, Y. X.; Lee, L. P. Nano Letters 2005, 5, 119-124. 
(24) Wu, L. Y.; Ross, B. M.; Lee, L. P. Nano Letters 2009, 9, 1956-1961.

(25) Unger, A.; Rietzler, U.; Berger, R.; Kreiter, M. Nano Letters 2009, 9, 2311-2315.

(26) Knight, M. W.; Halas, N. J. New Journal of Physics 2008, 10, 105006.

(27) Ross, B. M.; Lee, L. P. Nanotechnology 2008, 19, 275201.

(28) Prodan, E.; Radloff, C.; Halas, N. J.; Nordlander, P. Science 2003, 302, 419-422.

(29) Pendry, J. B.; Aubry, A.; Smith, D.; Maier, S. A. Science 2012, 337, 549-552.

(30) Aubry, A.; Lei, D. Y.; Fernández-Domínguez, A. I.; Sonnefraud, Y.; Maier, S. A.; Pendry, J. B. Nano Letters 2010, 10, 2574-2579.

(31) Luo, Y.; Pendry, J. B.; Aubry, A. Nano Letters 2010, 10, 4186-4191.

(32) Fernández-Domínguez, A. I.; Maier, S. A.; Pendry, J. B. Physical Review Letters 2010, 105, 266807.

(33) Luo, Y.; Lei, D. Y.; Maier, S. A.; Pendry, J. B. Physical Review Letters 2012, 108, 023901.

(34) Luo, Y.; Lei, D. Y.; Maier, S. A.; Pendry, J. B. ACS Nano 2012, 6, 6492-6506.

(35) Aubry, A.; Lei, D. Y.; Maier, S. A.; Pendry, J. B. Physical Review B 2010, 82, 205109.

(36) Wiener, A.; Fernández-Domínguez, A. I.; Horsfield, A. P.; Pendry, J. B.; Maier, S. A. Nano Letters 2012, 12, 3308-3314.

(37) Toscano, G.; Raza, S.; Jauho, A.-P.; Mortensen, N. A.; Wubs, M. Optics Express 2012, 20, 4176-4188.

(38) Johnson, P. B.; Christy, R. Physical Review B 1972, 6, 4370-4379.

(39) Aubry, A.; Lei, D. Y.; Maier, S. A.; Pendry, J. B. Physical Review B 2010, 82, 125430.

(40) Fernández-Domínguez, A. I.; Maier, S. A.; Pendry, J. B. Physical Review B 2012, 85, 165148. 
(41) David, C.; García de Abajo, F. J. Journal of Chemistry C 2011, 115, 19470 âĂŞ19475.

(42) Esteban, R.; Borisov, A. G.; Nordlander, P.; Aizpurua, J. Nature Materials 2012, 11, 573584.

(43) Scholl, J. A.; Koh, A. L.; Dionne, J. A. Nature 2012, 483, 421-427.

(44) Ciraci, C.; Hill, R. T.; Mock, J. J.; Urzhumov, Y.; Fernández-Domínguez, A. I.; Maier, S. A.; Pendry, J. B.; Chilkoti, A.; Smith, D. R. Science 2012, 337, 1072-1074.

(45) Fernández-Domínguez, A. I.; Wiener, A.; García-Vidal, F. J.; Maier, S. A.; Pendry, J. B. Physical Review Letters 2012, 108. 


\section{Graphical TOC Entry}

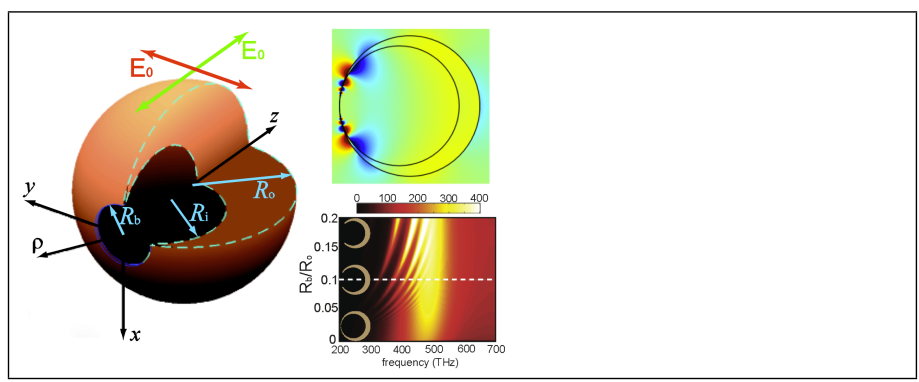

